\title{
Article
}

\section{Predicting functional outcome of ischemic stroke patients in Romania based on plasma CRP, sTNFR-1, D-Dimers, NGAL and NSE measured using a biochip array}

\author{
Adina HUȚANU ${ }^{1,2, \#, *}$, Mihaela IANCU ${ }^{3, \#, ~ R o d i c a ~ B A ̆ L A S ̦ A ~}{ }^{4,5}$, Smaranda MAIER ${ }^{4,5}$, Minodora DOBREANU ${ }^{2,6}$ \\ ${ }^{1}$ Laboratory Medicine, Emergency Clinical County Hospital, Tirgu Mures, Romania; ${ }^{2}$ Advanced Medical and Pharmaceutical \\ Research Center, University of Medicine and Pharmacy Tîrgu Mures, Tîrgu Mures, Romania; ${ }^{3}$ Department of Medical Informatics and \\ Biostatistics, University of Medicine and Pharmacy "Iuliu Hatieganu”, Cluj Napoca, Romania; ${ }^{4}$ First Neurological Clinic, Emergency \\ Clinical County Hospital, Tirgu Mures, Romania; ${ }^{5}$ Department of Neurology, University of Medicine and Pharmacy; ${ }^{6}$ Department of \\ Laboratory Medicine, University of Medicine and Pharmacy, Tirgu Mures, Romania
}

\begin{abstract}
In cerebral ischemia, evaluation of multiple biomarkers involved in various pathological pathways is a useful tool in assessing the outcome of the patients even from the early stages of the disease. In this study we investigated the utility of a panel of 5 peripheral biomarkers of inflammatory status, neuronal destruction and secondary fibrinolysis in the acute phase of ischemia, and evaluated the impact of these biomarkers on functional outcome after ischemic stroke. The 5 biomarkers (plasma CRP, D-Dimers, sTNFR-1, NGAL and NSE) were measured using a biochip array technology. Eighty nine patients in Romania were divided into 2 subgroups using the modified Rankin Scale evaluated at 3 months after ischemic stroke; the possible impact of analyzed biomarkers on unfavorable functional outcome was tested by binomial logistic regression. The subgroup with unfavorable outcome had higher concentrations of CRP, NGAL, STNFR-1 and D-dimers, but CRP and NGAL values were not statistically different between the two subgroups. The univariate logistic regression analysis of plasma biomarkers revealed that CRP, D-Dimers, NGAL, sTNFR-1 were significant predictors of unfavorable clinical outcome. In the case of D-Dimers and STNFR-1 we noticed an increased discrimination ability (versus baseline clinical model) to classify poor functional outcome with a tendency toward statistical signification. During the acute phase of the ischemic stroke, plasma concentrations of CRP, D-Dimers and STNFR-1 were elevated in unfavorable outcome patients. D-Dimers and STNFR-1 were independent predictors of poor outcome at 3 months after ischemic stroke. The biochip array technology offers the possibility to simultaneously measure several parameters involved in multiple pathophysiological pathways, in a small sample volume.
\end{abstract}

Keywords: stroke; biomarkers; C reactive protein (CRP); the soluble receptor 1 of TNF alfa (sTNFR-1); D-Dimers; neuron-specific enolase (NSE); neutrophil-gelatinase associated-lipocalin (NGAL); biochip array

Acta Pharmacologica Sinica (2018) 39: 1228-1236; doi: 10.1038/aps.2018.26; published online 7 Jun 2018

\section{Introduction}

Among non-communicable disorders, stroke is one of the most prevalent causes of death especially in developed countries ${ }^{[1]}$, ranking the third common cause of disability-adjusted lifeyears (DALYs) ${ }^{[2]}$, being an enormous burden for society as the stroke was no longer considered as a disease of old age ${ }^{[3]}$. As the global life expectancy increases for both sexes, the death

\footnotetext{
"The first two authors contributed equally to this work.

* To whom correspondence should be addressed.

E-mail adina.hutanu@umftgm.ro

Received 2017-11-04 Accepted 2018-02-19
}

rate also recorded an increase during the same period, from 47.5 million to 54.9 million, but the causes of death are different among low- and high-income counties. Given the high mortality rate and the high degree of disability, it is important to identify as soon as possible a peripheral biomarker, as well as for the possible hemorrhagic transformation of ischemic stroke. A comprehensive review about peripheral biomarkers and their importance in establishing the diagnosis, type of stroke, severity and prognosis, or the response to a therapy has been published recently ${ }^{[4]}$. Isolated biomarkers may confer truncate information about ischemic stroke, but evaluation of multiple biomarkers simultaneously could provide additional 
valuable information, with greater sensitivity and specificity than a single marker assessment.

C Reactive Protein (CRP) is a proinflammatory protein produced by the liver under pro-inflammatory cytokine induction in acute-phase response. Elevated levels of high sensitive CRP (hsCRP) are associated both with an increased risk of ischemic stroke $^{[5]}$ as well as with poor functional outcome and recurrent stroke prediction in patients with minor stroke or transient ischemic attack (TIA) ${ }^{[6]}$.

Neutrophil-gelatinase associated-lipocalin (NGAL) or Lipocalin 2 (LCN2) is an acute-phase reactant, released in particular by neutrophil granules, but also to a small extent from epithelial and macrophage cells, during the inflammatory response, involved in apoptosis mediated by intracellular iron chelation ${ }^{[7]}$ and additionally promoting the in vivo cell migration ${ }^{[8]}$.

sTNFR-1 the soluble receptor 1 of TNF alfa (Tumor Necrosis Factor alfa), is an important player during the oxygen-glucose deprivation, being involved in apoptosis after caspase cascade activation $^{[9]}$ but also modulating the neuroprotection exerted by EPO (erythropoietin) and VEGF (vascular endothelial growth factor $)^{[10]}$.

D-Dimers are markers of secondary fibrinolysis, plasma concentration was correlated with infarction volume in ischemic stroke patients, being predominantly elevated in cardioembolic (CE) stroke type ${ }^{[11]}$. The isoforms $\gamma \gamma$ - and a ${ }^{\text {-dimers of }}$ Neuron Specific Enolase (NSE) are very specific for the brain tissue. This biomarker is also secreted by several neuroendocrine tumors $^{[12]}$, so the sensitivity is still very low, especially when over ischemic pathology overlaps a neuroendocrine malignancy. Several studies have found that NSE concentration correlates with stroke severity at admission ${ }^{[13,14]}$ and with functional outcome evaluated after 30 days ${ }^{[15]}$, the concentration being a potential predictor of the outcome after recanalization therapy ${ }^{[16]}$.

There is no validated peripheral biomarker for stroke diagnosis and prognosis yet, so this subject is still opened for investigation on multiple pathways involved in the pathophysiology of stroke-inflammation, coagulation disorders, neuronal injury or Blood Brain Barrier (BBB) alteration. Previous studies have reported the importance of peripheral biomarkers in prediction of severity and functional outcome after ischemic stroke, most of them being analyzed separately ${ }^{[17-19]}$. Advances in technologies provide possibilities to study multiple biomarkers simultaneously in a panel, allowing quantification of several proteins in a small volume of serum or plasma sample, thus being more time and cost-effective.

The aim of the study was to investigate the utility of a panel of five peripheral biomarkers of inflammatory status, neuronal destruction and secondary fibrinolysis in the acute phase of ischemia and prediction of functional outcome at 3 months after discharge.

\section{Materials and methods}

\section{Patients and study design}

During October 2014 and October 2015, we prospectively enrolled 124 patients with ischemic stroke diagnosis who were admitted within $72 \mathrm{~h}$ from the disease onset, in the First Department of Neurology, Emergency Clinical County Hospital Tîrgu Mures; 116 of them have fulfilled the inclusion criteria. The study was conducted according to the Declaration of Helsinki and the study protocol was approved by the Institutional Ethical Committee. Exclusion criteria were: age below 18 , history of stroke in the last 3 months, other concomitant inflammatory diseases and neurological disorders.

\section{Clinical data}

After admission, all stroke patients underwent a neurological examination; the neurological deficit was assessed by National Institute of Health Stroke Scale (NIHSS) on admission and periodically during hospitalization, being dichotomised in mild stroke (NIHSS $\leq$ ) and moderate/severe stroke (NIHSS $>7$ ). Functional outcome was evaluated by modified Rankin Scale (mRS) at discharge and 3 months afterward (data available only for 89 patients); a mRS of 0-2 points was defined as a good outcome, while a mRS of 3-6 points indicated poor outcome. Ischemic stroke subtype was classified using TOAST (Trial of Org 10172 in Acute Stroke Treatment) criteria in large artery atherosclerosis (LAA), cardioembolic (CE), small vessels occlusion (SVO), and other causes ${ }^{[20]}$. Doppler sonography was performed only in patients with LAA stroke type, while CE type was considered in patients with at least one possible source of emboli, the most common cause being predominantly atrial fibrillation.

\section{Blood sample collection and biomarkers measurement}

Blood was collected during the first morning after admission, in a Na-heparin blood collection tubes, immediately centrifuged at $1500 \times g$ for $15 \mathrm{~min}$ at $4{ }^{\circ} \mathrm{C}$; and plasma was aliquoted and stored at $-80{ }^{\circ} \mathrm{C}$ until analyses. Based on biochip array technology (BAT) from Randox Cerebral Array II (Randox Laboratories Limited, UK) we analyzed simultaneously five biomarkers: three inflammatory related biomarkers (CRP, NGAL and sTNFR-1), a marker of secondary fibrinolysis (D-Dimers) and a marker of neuronal destruction (neuronspecific enolase NSE). Measuring intervals were: 0-12 mg/L for CRP, 0-200 ng/mL for NSE, 0-2000 ng/mL for NGAL, 0-50 ng/mL for sTNFR1 and 0-2000 $\mathrm{ng} / \mathrm{mL}$ for D-Dimers. The chip sensitivities were: $0.67 \mathrm{mg} / \mathrm{L}$ for CRP, $17.80 \mathrm{ng} /$ $\mathrm{mL}$ for NGAL, $0.24 \mathrm{ng} / \mathrm{mL}$ for sTNFR-1, $2.10 \mathrm{ng} / \mathrm{mL}$ for D-Dimers and $0.26 \mathrm{ng} / \mathrm{mL}$ for NSE. We performed also hsCRP analysis by enhanced immunoturbidimetric method (hsCRP vario, Architect 4000 Abbott Laboratories), limit of detection: $0.1 \mathrm{mg} / \mathrm{L}$, measuring range $0.1-160 \mathrm{mg} / \mathrm{L}$, within- and interassay precision $<4 \%$.

\section{Statistical analysis}

Statistical analysis of data was released with the statistical advanced environment R (v.3.4.0, Vienna, Austria). To assess the normality of continuous variables (i.e. CRP, NGAL, sTNFR-1, D-Dimers and NSE), the Shapiro-Wilk test and point and confidence interval estimation for univariate skewness and kurtosis were applied. Student's $t$-test was used to 
assess the differences between means of continuous variables (expressed as mean $\pm S D$ ), while differences between distributions of nonparametric variables were evaluated using the Mann-Whitney test. To analyze the associations between qualitative variables we used contingency tables and the chisquare or exact Fisher test.

The possible impact of plasma markers in unfavorable functional outcome at three months was tested by binomial logistic regression and the strength of association was estimated by crude and adjusted OR (odds ratio) and 95\% CI associated. Because the panel of biomarkers was determined for 56 patients, we used the imputation method by means or medians for missing data in order to have the possibility of using all available clinical information.

A predictor was defined as a significant independent predictor when the $95 \%$ CI for adjusted OR did not cross reference value $(=1)$.

All above-described statistical methods had tested associations between peripheral biomarkers and the probability (odds) of an unfavorable functional outcome at 3 months. Thereby, each biomarker has been studied both individually and in relation to the known risk factors of the poor outcome of the ischemic event.

A separate logistic regression was performed for each parameter of interest running 3 additive models: Model 1 including each of the biochip parameters and demographic variables as age and gender, Model 2 considered as baseline clinical model including all variables of Model 1 plus stroke severity measured by initial NIHSS score and Model 3 including all variables of Model 2 plus known clinical variables. Because of the relatively small sample size, the known clinical factors were the variables having a minimum frequency of 5 cases for the event (unfavorable functional outcome) and an estimated significance level in the univariate regression analysis $P \leq 0.25^{[21]}$. In all models, the five peripheral biomarkers were transformed into categorical variables (using tertiles) in order to highlight the magnitude of the values for studied parameters.

Because of the small sample size, the combined effect of biomarkers on the unfavorable outcome of ischemic stroke was investigated, but it could not be adjusted depending on the presence of covariates used in previous models (age, sex, stroke severity, dyslipidemia, atrial fibrillation and history of stroke).

The capacity of discrimination between patients with unfavorable and favorable outcome was determined by c-statistics by calculating the area under ROC curve (AUC) for each of the model tested.

For all bilateral tests, a statistical significance was considered when $P$-values were lower than significance threshold $(a=0.05)$.

\section{Results}

\section{Description of the sample}

A number of 89 subjects from eligible enrolled patients were further studied. We divided patients into 2 subgroups accordingly: patients with mRS score 0,1 and 2 evaluated at three months were considered as having favorable functional outcome and patients with mRS score greater than 3 as having unfavorable (poor) functional outcome. As expected, the unfavorable outcome subgroup had a higher score for initial NIHSS scale than the favorable outcome subgroup $(P<0.001$, Supplementary Table S1). There was no significant association between TOAST classification and unfavorable clinical outcome $(P=0.288)$. The demographic and laboratory variables were described in Table 1 . The age of studied patients was $71.9 \pm 10$ years. There was a significant difference in age distribution between unfavorable and favorable outcome subgroups (Student $t$-test, $t(87)=4.45, P<0.001$ ) while gender distribution in poor and favorable outcome subgroups was similar (Chi-square test, $\chi 2(1)=2.39, P=0.145)$.

The mean or median concentrations of biochip panel markers are presented in Table 2. The median and interquartile range of time after stroke onset was $10 \mathrm{~h}^{[7-12]}$ in the studied sample.

The subgroup with unfavorable outcome had higher concentrations of CRP, hsCRP, NGAL, sTNFR-1 and D-Dimers (Table 2), but CRP and NGAL were not statistically different between the two subgroups.

Because the panel of biochip markers was determined only for 56 patients, the missing values of parameters were replaced by the mean of observed values (in the case of CRP, NSE, NGAL) in each subgroup or by median value (case of D-Dimers and sTNFR-1). Replacing missing values by these strategies no significant differences were found between patients with complete data on biochip parameters and patients with missing data (Table 3). Patients with missing biomarkers values did not differ with respect to age, gender and stroke severity from patients with measured biomarkers.

The univariate logistic regression analysis of chip plasma biomarkers revealed that CRP, D-Dimers, NGAL, sTNFR-1 were significant predictors of unfavorable clinical outcome (Table 4). For the multivariate analysis using logistic regression, we tested three models in order to highlight the prognostic contribution of studied biomarkers to the clinical outcome. There were no multicollinearity problems between tested predictors. The statistical significance of CRP remained after controlling for age, gender and stroke severity (T3 vs T1: $\mathrm{OR}=4.42$, 95\% CI: 1.06-20.42), but in the presence of dyslipidemia, atrial fibrillation, and history of stroke there is a tendency towards statistical significance (T3 vs T1: OR=4.43, 95\% CI: 0.95-21.11). Adding CRP to the Model 1, increased the $-2 \log$ likelihood ratio (-2LLModel2=38.30 vs -2 LLModel1 $=29.56)$ and goodness of fit (Hosmer-Lemeshow's test, $P=0.336$ ) while adding known clinical factors to Model 2 increased the -2 log likelihood ratio (-2LLModel3=41.63 vs -2LLModel2=38.30) and goodness of fit (Hosmer-Lemeshow's test, $P=0.147$ ).

The D-Dimers remained significant predictor after controlling for age and gender and stroke severity (T3 vs T1: $\mathrm{OR}=10.40$, 95\% CI: 1.38-78.16)), dyslipidemia, atrial fibrillation, and history of stroke (T3 vs T1: OR=10.85, 95\% CI: 1.28-91.84). Adding D-Dimers to the Model 1, increased the 
Table 1. The distribution of demographic, clinical and laboratory characteristics in all sample and studied subgroups.

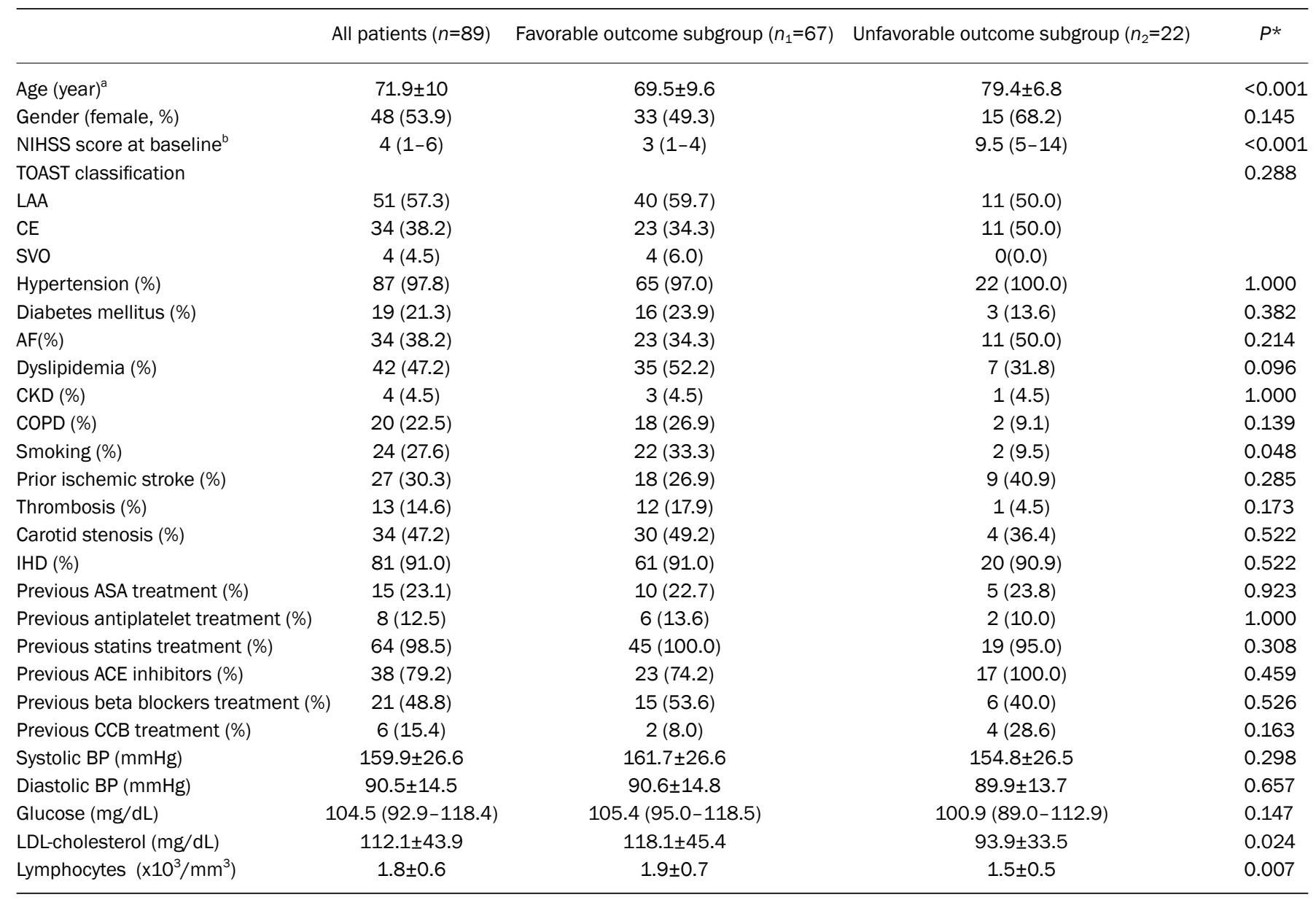

${ }^{a}$ Mean \pm standard deviation; ${ }^{b}$ Median (interquartile interval:percentile 25\%-percentile 75\%);

*Estimated significance level obtained from Student $t$-test, Mann-Whitney or Chi-square tests. AF: atrial fibrillation; CKD: Chronic kidney disease; COPD: chronic obstructive pulmonary disease; IHD: ischemic heart disease; ASA: acetylsalicylic acid; ACE: angiotensin-converting-enzyme; CCB: Calcium channel blockers; LDL; low-density lipoproteins; LAA: large-artery atherosclerosis; CE: cardioembolism; SVO: small-vessel occlusion.

-2 log likelihood ratio (-2LLModel2=41.62 vs -2LLModel1=29.56) and goodness of fit (Hosmer-Lemeshow's test, $P=0.948$ ), while adding known clinical factors to the Model 2, increased the -2 $\log$ likelihood ratio (-2LLModel3 $=45.13$ vs -2 LLModel2 $=41.62$ ) and goodness of fit (Hosmer-Lemeshow's test, $P=0.664$ ).

The sTNFR-1 remained significant predictor after controlling for age and gender and stroke severity ( $\mathrm{T} 3$ vs $\mathrm{T} 1$ : $\mathrm{OR}=7.43$,
95\% CI: 1.12-49.18), dyslipidemia, atrial fibrillation, and history of stroke (T3 vs T1: OR=7.16, 95\% CI: 1.03-49.76). Adding sTNFR-1 to the Model 1, increased the $-2 \log$ likelihood ratio (-2LLModel2 $=40.86$ vs -2 LLModel1 $=29.56)$ and goodness of fit (Hosmer-Lemeshow's test, $P=0.547$ ) while adding known clinical factors to the Model 2, increased the $-2 \log$ likelihood ratio $(-2$ LLModel3 $=44.28$ vs -2 LLModel2 $=40.86)$ and goodness

Table 2. Comparison of plasmatic concentrations on chip biomarkers between studied subgroups.

\begin{tabular}{lccc}
\hline Laboratory parameters & All patients $(n=89)$ & Favorable outcome subgroup $\left(n_{1}=67\right)$ & Unfavorable outcome subgroup $\left(n_{2}=22\right)$ \\
\hline CRP $(\mathrm{mg} / \mathrm{L})$ & $6.3 \pm 3.23$ & $6.0 \pm 3.22$ & $7.4 \pm 3.09$ \\
hsCRP $(\mathrm{mg} / \mathrm{L})$ & $5.0(3.00-12.00)$ & $5.0(2.00-10.00)$ & $9.0(4.00-14.00)$ \\
NGAL $(\mathrm{ng} / \mathrm{mL})$ & $742.4 \pm 255.91$ & $729.7 \pm 256.33$ & $781.7 \pm 256.60$ \\
NSE $(\mathrm{ng} / \mathrm{mL})$ & $15.9 \pm 8.81$ & $17.2 \pm 7.28$ & $12.3 \pm 4.89$ \\
STNFR1 $(\mathrm{ng} / \mathrm{mL})$ & $1.1(0.90-1.31)$ & $1.1(0.83-1.12)$ & $1.5(1.15-1.52)$ \\
D Dimers $(\mathrm{ng} / \mathrm{mL})$ & $185.1(185.06-245.06)$ & $185.1(166.33-203.61)$ & $245.1(230.09-331.20)$ \\
\hline
\end{tabular}

*Estimated significance level obtained from Student $t$-test, Mann-Whitney or Chi-square tests. 
Table 3. The analysis of missing values effect on demographic and clinical characteristics.

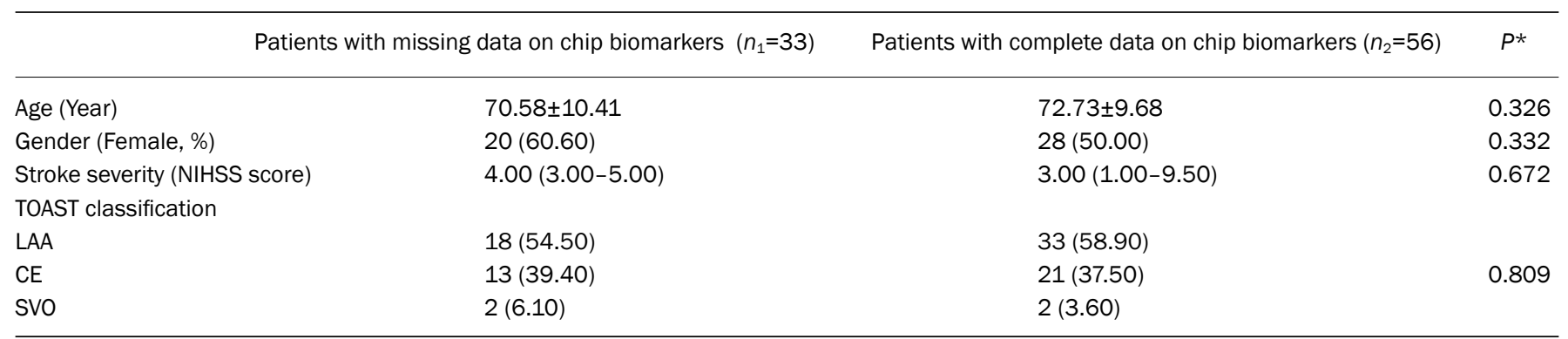

*Estimated significance level obtained from Student $t$-test, Mann-Whitney or Chi-square tests.

of fit (Hosmer-Lemeshow's test, $P=0.099)$.

The NGAL remained a predictor with tendency toward statistical significance after adjusting for age, gender and stroke severity (T3 vs T1: OR=3.20, 95\% CI: 0.70-14.58) and it not remained a significant predictor in the presence of dyslipidemia, atrial fibrillation, and history of stroke (T3 vs T1: OR=3.10, 95\% CI: 0.65-14.68). Adding NGAL to the Model 1, increased the $-2 \log$ likelihood ratio (-2LLModel2 $=38.14$ vs -2 LLModel1=29.56) and goodness of fit (HosmerLemeshow's test, $P=0.533)$ while adding known clinical factors to the Model 2, increased the $-2 \log$ likelihood ratio (-2LLModel3=42.07 vs -2LLModel2=38.14).

We tested in the ROC analysis the effect of the addition of each biomarker on the baseline clinical model for the poor outcome prediction (Figure 1).
Adding CRP biochip marker the predictive capacity of baseline clinical model was improved but not significant ( $P=0.153$ by DeLong test $): A U C=0.902,95 \%$ CI: $0.837-0.966$ versus $A U C=0.856,95 \%$ CI: 0.771-0.940 without CRP. Adding D-Dimer biochip marker the predictive capacity of baseline clinical model was improved with a tendency toward statistical signification $(P=0.088$ by DeLong test): $A U C=0.913,95 \%$ CI: 0.856-0.970. Adding NGAL biochip marker the predictive capacity of baseline clinical model was improved without statistical signification ( $P=0.173$ by DeLong test): $A U C=0.907$, 95\% CI: 0.832-0.960. Adding sTNFR-1 biochip marker the predictive capacity of baseline clinical model was improved with a tendency toward statistical signification $(P=0.079$ by DeLong test): $\mathrm{AUC}=0.913,95 \% \mathrm{CI}: 0.844-0.970$.

Table 4. Univariate and multivariate associations of biochip array markers with functional outcome at 3 months in patients with ischemic stroke.

\begin{tabular}{|c|c|c|c|c|c|}
\hline \multirow[t]{2}{*}{ Biomarker } & \multirow[t]{2}{*}{ Model } & \multicolumn{3}{|c|}{ OR (95\% Cl) } & \multirow[t]{2}{*}{$P$-value } \\
\hline & & Lower tertile (T1) & Middle tertile (T2) & Upper tertile (T3) & \\
\hline \multirow[t]{4}{*}{ CRP } & & $\leq 2.86$ & $2.86-9.48$ & $>9.48$ & \\
\hline & Unadjusted & 1 (Reference) & $0.33(0.06-1.85)$ & $4.31(1.30-14.27)$ & 0.002 \\
\hline & Model 2 & 1 (Reference) & $0.44(0.06-3.45)$ & $4.42(1.06-20.42)$ & 0.023 \\
\hline & Model 3 & 1 (Reference) & $0.62(0.07-5.30)$ & $4.48(0.95-21.11)$ & 0.046 \\
\hline \multirow[t]{4}{*}{ D-dimers } & & $\leq 170.84$ & $170.84-246.93$ & $>246.93$ & \\
\hline & Unadjusted & 1 (Reference) & $1.21(0.20-7.23)$ & $11.43(2.26-57.80)$ & $<0.001$ \\
\hline & Model 2 & 1 (Reference) & $1.06(0.12-9.46)$ & $10.40(1.38-78.16)$ & 0.007 \\
\hline & Model 3 & 1 (Reference) & $1.12(0.11-11.22)$ & $10.85(1.28-91.84)$ & 0.015 \\
\hline \multirow[t]{4}{*}{ NSE } & & $\leq 10.01$ & $10.01-16.83$ & $>16.83$ & \\
\hline & Unadjusted & 1 (Reference) & $0.57(0.20-1.66)$ & $0.43(0.10-1.89)$ & 0.438 \\
\hline & Model 2 & 1 (Reference) & $2.53(0.52-12.31)$ & $0.98(0.11-8.94)$ & 0.448 \\
\hline & Model 3 & 1 (Reference) & $2.27(0.44-11.83)$ & $0.60(0.05-6.55)$ & 0.418 \\
\hline \multirow[t]{4}{*}{ NGAL } & & $\leq 566.43$ & $566.43-853.86$ & $>853.86$ & \\
\hline & Unadjusted & 1 (Reference) & $0.11(0.02-0.95)$ & $2.75(0.90-8.37)$ & 0.006 \\
\hline & Model 2 & 1 (Reference) & $0.20(0.02-2.28)$ & $3.20(0.70-14.58)$ & 0.050 \\
\hline & Model 3 & 1 (Reference) & $0.21(0.02-2.62)$ & $3.10(0.65-14.68)$ & 0.071 \\
\hline \multirow[t]{4}{*}{ sTNFR-1 } & & $\leq 0.82$ & $0.82-1.21$ & $>1.21$ & \\
\hline & Unadjusted & 1 (Reference) & $0.45(0.08-2.65)$ & 7.14 (1.99-25.59) & $<0.001$ \\
\hline & Model 2 & 1 (Reference) & $0.47(0.06-3.95)$ & $7.43(1.12-49.18)$ & 0.012 \\
\hline & Model 3 & 1 (Reference) & $0.50(0.06-4.57)$ & $7.16(1.03-49.76)$ & 0.024 \\
\hline
\end{tabular}

Model 2: controlling (adjusting) for age, gender and stroke severity (initial NIHSS scores were dichotomized in NIHSS>7 and NIHSS $\leq 7$ points); Model 3: adjusted for variables from Model 2 plus dyslipidemia, atrial fibrillation and history of stroke. 


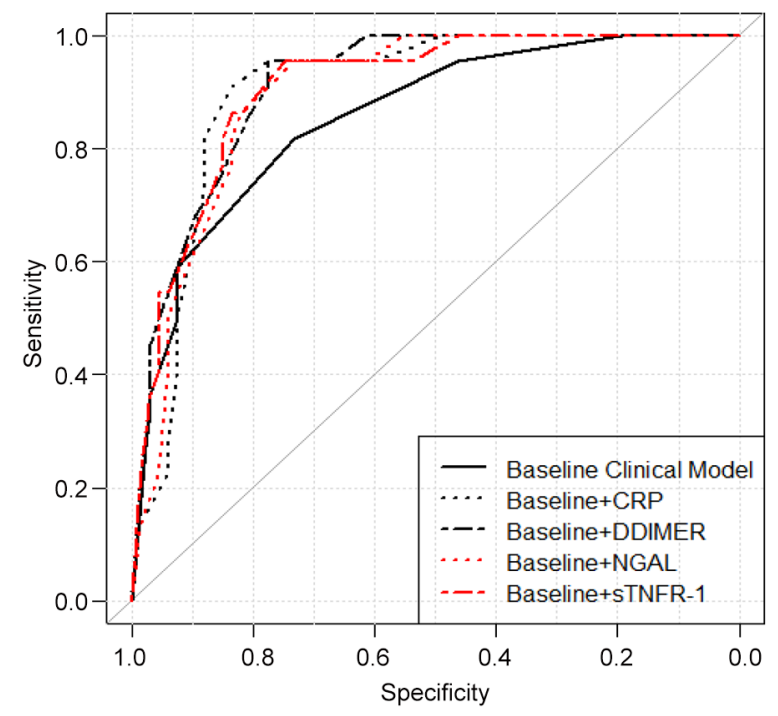

Figure 1. The ROC curves of each model containing studied biochip array marker.

\section{Combined associations}

When biomarkers, identified as significant in separate unadjusted models, were included in a multivariate model, only D-Dimer was an independent predictor significantly related to the risk of unfavorable outcome of ischemic stroke (Table 5 ), the upper tertile of D-Dimers (values of D-dimers $>246.93$ $\mathrm{ng} / \mathrm{mL}$ ) being a risk factor for unfavorable outcome.

hsCRP was not found as significant predictor of unfavorable outcome after ischemic stroke (Likelihood Ratio test, $P=0.244$ ).

The model containing multiple biomarkers together had a better discrimination than models containing any individual stroke poor outcome independent predictor, like CRP $(P=0.011)$, D-Dimer $(P=0.019)$, NGAL $(P=0.007)$ and sTNFR-1
$(P=0.031)$ (Table 6).

Figure 2 highlights that chip biomarkers (CRP, D-dimers, NGAL and sTNFR-1) taken together give greater sensitivity and specificity than each individual biomarker.

\section{Discussion}

In our study, the biochip assay technology allowed us a simultaneous evaluation of multiple biomarkers involved in different pathways of cerebral ischemic stroke event. Inflammatory markers, along with markers of leucocyte activation, secondary fibrinolysis and neuronal destruction were tested on a panel, based on biochip array technology. Our results revealed that some of the studied biomarkers were predictive for 3 months poor outcome evolution. Because none of the studied biomarkers is very specific for stroke, the selection of the patients was very rigorous.

CRP as well as highly sensitive isoform-hsCRP is a very well studied inflammatory marker in cardio-cerebrovascular diseases. Increased concentration within $12 \mathrm{~h}$ after symptoms onset of ischemic stroke is an independent prognostic factor of poor outcome at three months ${ }^{[22]}$. Additionally, CRP level at admission was associated with early neurological deterioration in acute ischemic stroke patients ${ }^{[23]}$. In our study higher levels $(9.48-12 \mathrm{mg} / \mathrm{L})$ of CRP were predictive for unfavorable clinical outcome after controlling for age, gender and stroke severity, but in the presence of dyslipidemia, atrial fibrillation or history of stroke there was only a tendency towards statistical significance.

NGAL as an acute-phase reactant is released in particular by neutrophil granules during the inflammatory response. An experimental study analyzing the co-expression of specific antibodies for NGAL and neutrophils, neurons, microglia and astrocytes revealed that NGAL is predominantly co-expressed in blood-derived neutrophils in the peri-infarct region and in astrocytes $\left(\mathrm{GFAP}^{+}\right)$in the ipsilateral hemisphere, but not in

Table 5. Multiple biomarkers logistical model for unfavorable outcome prediction at three months after ischemic stroke.

\begin{tabular}{|c|c|c|}
\hline Biomarkers & Adjusted Odds ratio $(95 \% \mathrm{Cl})$ & $P$-value \\
\hline CRP & & 0.767 \\
\hline CRP lower tertile (below 2.86 mg/L) & Reference category & Reference category \\
\hline CRP middle tertile (2.86 to $9.48 \mathrm{mg} / \mathrm{L}$ ) & $0.74(0.06-9.39)$ & 0.813 \\
\hline CRP upper tertile (above 9.48 mg/L) & $1.55(0.33-7.39)$ & 0.582 \\
\hline D-dimers & & 0.045 \\
\hline D-dimers lower tertile (below 170.84 ng/mL) & Reference category & Reference category \\
\hline D-dimers middle tertile (170.84 to $246.93 \mathrm{ng} / \mathrm{mL}$ ) & $3.39(0.35-32.81)$ & 0.292 \\
\hline D-dimers upper tertile (above 246.93 ng/mL) & $8.36(1.47-47.62)$ & 0.017 \\
\hline$N G A L$ & & 0.333 \\
\hline NGAL lower tertile (below 566.43 ng/mL) & Reference category & Reference category \\
\hline NGAL middle tertile (566.43 to $853.86 \mathrm{ng} / \mathrm{mL}$ ) & $0.09(0.01-2.17)$ & 0.139 \\
\hline NGAL upper tertile (above 853.86 ng/mL) & $0.79(0.15-4.18)$ & 0.784 \\
\hline sTNFR-1 & & 0.260 \\
\hline sTNFR-1 lower tertile (below 0.82 ng/mL) & Reference category & Reference category \\
\hline sTNFR-1 middle tertile (0.82 to $1.21 \mathrm{ng} / \mathrm{mL}$ ) & $2.60(0.18-37.58)$ & 0.483 \\
\hline sTNFR-1 upper tertile (above $1.21 \mathrm{ng} / \mathrm{mL}$ ) & $4.92(0.73-32.99)$ & 0.101 \\
\hline
\end{tabular}


Table 6. Discrimination ability of multiple biomarkers models for poor outcome prediction at three months after ischemic stroke.

\begin{tabular}{lcc}
\hline Models & AUC $(95 \% \mathrm{Cl})$ & $P^{\text {-value }}{ }^{*}$ \\
\hline CRP & $0.753(0.646-0.860)$ & -- \\
+ D-dimers & $0.828(0.726-0.930)$ & 0.098 \\
+ NGAL & $0.803(0.694-0.912)$ & 0.326 \\
+ sTNFR-1 & $0.833(0.750-0.916)$ & 0.039 \\
+ D-dimers+NGAL+sTNFR-1 & $0.876(0.805-0.946)$ & 0.011 \\
D-dimers & $0.764(0.651-0.877)$ & -- \\
+ NGAL & $0.850(0.770-0.929)$ & 0.062 \\
+ sTNFR-1 & $0.853(0.774-0.932)$ & 0.062 \\
+ CRP+NGAL+sTNFR-1 & $0.876(0.805-0.946)$ & 0.019 \\
NGAL & $0.760(0.662-0.858)$ & -- \\
+ sTNFR-1 & $0.808(0.711-0.905)$ & 0.298 \\
+ CRP+D-dimers+sTNFR-1 & $0.876(0.805-0.946)$ & 0.007 \\
sTNFR-1 & $0.780(0.675-0.886)$ & -- \\
+ CRP+NGAL+sTNFR-1 & $0.876(0.805-0.946)$ & 0.031 \\
\hline
\end{tabular}

*Observed significance levels obtained from DeLong test for comparison of AUCs.

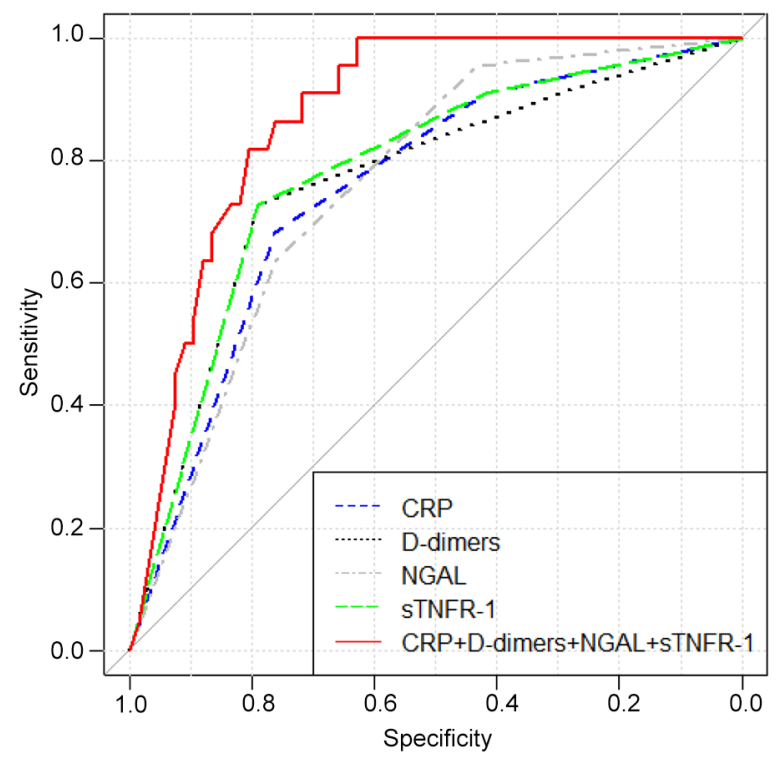

Figure 2. CRP, D-dimers, NGAL and STNFR-1 taken together give greater sensitivity and specificity than each individual biomarker.

neurons $\left(\mathrm{NeuN}^{+}\right)$and Iba1 positive microglia ${ }^{[24]}$. Additionally, NGAL expression was significantly lower in pMCAO (permanent Middle Cerebral Artery Occlusion) than in tMCAO (transient Middle Cerebral Artery Occlusion), suggesting that reperfusion is another aggravating factor of ischemic injury ${ }^{[24]}$. NGAL has the ability to covalently bound to MMP-9 (Matrix Metalloproteinase 9) involved in BBB destruction during ischemia, thus preventing degradation and preserving its gelatinolytic activity ${ }^{[25]}$, the concentration of the NGAL-MMP-9 heterodimers complex being higher in hemorrhagic than in ischemic stroke patients ${ }^{[26]}$. Plasma level of NGAL was associated with the occurrence of post-stroke infections and implicited worse clinical outcome at 90 days $^{[27]}$. Our results are in line with the data from the literature, plasma level of NGAL was higher in poor outcome patients compared to good outcome patients and was a predictor with tendency toward statistical significance after adjusting for age, gender and stroke severity but lost the significance in the presence of dyslipidemia, atrial fibrillation, and history of stroke.

NSE could be considered a surrogate marker for neuronal destruction, also secreted by several neuroendocrine tumors. A recent comprehensive review of the literature regarding the peripheral biomarkers for brain injury highlighted the importance of NSE in neuronal function and the meaning of increased plasma levels after different brain injury-traumatic or ischemic-as well as in other pathological conditions as bone fracture, kidney or liver damage and migraine, so limiting its utility as a neuron-specific marker ${ }^{[28]}$. A recent study found elevated NSE concentration up to 5 months after ischemic stroke compared to controls, with higher serum levels in severe neurologic impairment, and in patients with concomitant diabetes mellitus or ischemic heart disease ${ }^{[29]}$. During the acute episode of ischemic stroke, the peripheral levels of NSE correlate with infarct volume and functional outcome after 1 month $^{[15]}$ and with stroke severity on admission ${ }^{[30]}$. In the present study, in contradiction with data from the literature, there was no difference between ischemic stroke patients and controls, and the levels were significantly higher in patients with a favorable outcome. Additionally, NSE was not prefigured as a predictive marker of functional outcome for patients' evaluation at three months.

D-Dimers, a marker of secondary fibrinolysis, was found to correlate with stroke severity and ischemic volume, independent of other variables ${ }^{[11]}$, as well to be an independent predictor of poor prognosis ${ }^{[31]}$. A concentration of $1090 \mathrm{ng} / \mathrm{mL}$ was found as a cutoff value for CE stroke with $87.5 \%$ sensitivity and $76.5 \%$ specificity for discrimination between TOAST subtype $^{[32]}$. A research study on the implication of CRP in inflammation and coagulation pathways was accomplished in healthy male volunteers by recombinant human CRP administration. Aside the inflammatory response, the activation of the pro-coagulant response was associated with a 3 -fold increase in prothrombin concentration, a 3.5-fold increase of D-Dimers concentrations and augmented concentration of plasminogen activator inhibitor type- $1^{[33]}$. Additionally, fibrinogen degradation products stimulate IL-6 production independent of prostaglandin induction in isolated murine liver ${ }^{[34]}$. It may be speculated that D-Dimers implication in unfavorable evolution after ischemic stroke could be related to their concomitant inflammatory and pro-coagulant actions, but additional studies are needed to elucidate the exact mechanism of D-Dimers in unfavorable stroke prognosis. In our study, we did not find any difference between TOAST subtype of stroke regarding the D-Dimers concentration, probably due to small sample size, but higher levels $(>246.93 \mathrm{ng} / \mathrm{mL})$ of D-Dimers remained significant predictors after controlling for age, gender, stroke severity and other clinical factors, results which are in line 
with data from the literature.

TNF alfa is a pleiotropic cytokine with a biphasic secretion, with both neurotoxic and neuroprotective effects upon the nervous tissue during the ischemic episode, depending on the region of production, as was revealed in a recent review of the literature $^{[35]}$. During the ischemic attack, TNF alfa, produced locally mainly by the activated microglia and macrophages, enhances excitotoxicity, oxidative stress and neuronal vulnerability, also promoting the apoptosis of the endothelial cells and BBB destruction eventually leading to vasogenic edema ${ }^{[35]}$. Contrariwise, TNF alfa is involved in neuronal survival and plasticity, modulating the NF-kappaB pathway involved both in the apoptosis and cell protection ${ }^{[36]}$ and is responsible, via TNFR-1, for up-regulation of integrins expression involved in angiogenesis and tissue remodeling after ischemic stroke ${ }^{[37]}$. Literature review suggests that the detrimental or protective role of TNF alfa is region-specific and depends on the type of receptors involved in the cell signaling ${ }^{[35]}$. Some recent studies found an association between increased plasma level of TNF receptors and risk of intracerebral hemorrhage ${ }^{[38]}$, or suggest the predictive value of an increased level of sTNFR-1 in the progression to death of patients with minor stroke independently of clinical and demographic risk factors ${ }^{[39]}$, but there are scarce data regarding the plasma level of sTNFR-1 and outcome after ischemic stroke. In the present study, plasma level of sTNFR-1 was found to be elevated in poor functional outcome patients evaluated at three months and was a significant predictor of unfavorable outcome after adjusting for age, gender, stroke severity, dyslipidemia, atrial fibrillation or history of stroke.

Except for NSE, adding each of studied parameters to the baseline clinical model increased the goodness of model fit to data. In the case of D-Dimers and sTNFR-1, we noticed an increased discrimination ability (versus baseline clinical model) to classify poor functional outcome with a tendency toward statistical signification.

Although in our study the patients were very rigorously selected, there are some limitations: first, we performed only one single measurement of peripheral markers, without investigating the dynamic evolution after the ischemic stroke episode. Serial measurements could give the opportunity to investigate if persistent higher levels of biomarkers remained predictive for functional outcome. Second, the measurement of studied biomarkers was not done in the entire patients'group, therefore the results should be interpreted with caution. Third, because of the small sample size, we could adjust only for a limited number of predictors of functional outcome following ischemic stroke. Another aspect that needs to be highlighted is the potential limitation of the biochip for the acute phase of the ischemic stroke, the measuring range being not wide enough for this stage of disorders so that some biomarkers exceed the upper limit of the measuring interval.

Further studies are needed to identify independent biomarkers contribution when the biomarkers will be taken together in one single model in the presence of the established clinical factors.
The strength of the present work is the evaluation of plasma CRP, D-Dimers, NSE, NGAL and sTNFR-1 impact on functional outcome in ischemic stroke patients in the context of known clinical covariates.

\section{Conclusion}

In our study, the concentrations of plasma CRP, D-Dimers and sTNFR-1 during the acute phase of the ischemic episode were elevated in unfavorable outcome patients. The higher levels of plasma CRP, D-Dimers and sTNFR-1 were predictive for poor functional prognosis at 3 months, independently of known demographic factors (age, gender) and clinical factors (stroke severity, dyslipidemia, atrial fibrillation and history of stroke). The biochip array technology offers the possibility to simultaneously measure multiple parameters involved in different pathophysiological pathways, in a small sample volume, saving time and resources.

\section{Acknowledgements}

This work was supported by a grant of the Romanian National Authority for Scientific Research and Innovation, CNCS/CCCDI-UEFISCDI, project number PN-III-P2-2.1PED-2016-0734 within PNCDI III, contract No 155 PED/2017. We are grateful to the physicians, nurses and patients from the Department of the 1-st Neurological Clinic, Emergency Clinical County Hospital Tîrgu Mures who participated in our study.

\section{Author contribution}

Adina HUȚANU designed the study, collected samples/data, analyzed samples, drafted the manuscript; Mihaela IANCU analyzed/interpreted data, drafted the manuscript; Smaranda MAIER evaluated the patients, collected clinical data; Rodica BĂLAȘA revised the manuscript; Minodora DOBREANU designed the study, revised the manuscript.

\section{Supplementary information}

Supplementary information is available at the website of Acta Pharmacologica Sinica.

\section{References}

1 Christopher P, Murray JL. Global, regional, and national age-sex specific all-cause and cause-specific mortality for 240 causes of death, 1990-2013: a systematic analysis for the Global Burden of Disease Study 2013. Lancet 2015; 385: 117-71.

2 Murray CJL, Vos T, Lozano R, Naghavi M, Flaxman AD, Michaud C, et al. Disability-adjusted life years (DALYs) for 291 diseases and injuries in 21 regions, 1990-2010: a systematic analysis for the Global Burden of Disease Study 2010. Lancet 2012; 380: 2197-223.

3 Feigin VL, Forouzanfar MH, Krishnamurthi R, Mensah GA, Connor $\mathrm{M}$, Bennett DA, et al. Global and regional burden of stroke during 1990-2010: Findings from the Global Burden of Disease Study 2010. Lancet 2014; 383: 245-54.

4 Maestrini I, Ducroquet A, Moulin S, Leys D, Cordonnier C, Bordet R. Blood biomarkers in the early stage of cerebral ischemia [Internet]. Vol. 172, Revue Neurologique; 2016. p198-219.

5 Zhou Y, Han W, Gong D, Man C, Fan Y. Hs-CRP in stroke: a meta- 
analysis. Clin Chim Acta 2016; 453: 21-7.

6 Li J, Zhao X, Meng X, Lin J, Liu L, Wang C, et al. High-sensitive C-reactive protein predicts recurrent stroke and poor functional outcome. Stroke 2016; 47: 2025-30.

7 Devireddy LR, Gazin C, Zhu X, Green MR. A cell-surface receptor for lipocalin $24 p 3$ selectively mediates apoptosis and iron uptake. Cell 2005; 123: 1293-305.

8 Lee S, Kim JH, Kim JH, Seo JW, Han HS, Lee WH, et al. Lipocalin-2 is a chemokine inducer in the central nervous system: role of chemokine ligand 10 (CXCL10) in lipocalin-2-induced cell migration. J Biol Chem 2011; 286: 43855-70.

9 Kogo J, Takeba Y, Kumai T, Kitaoka Y, Matsumoto N, Ueno S, et al. Involvement of TNF- $\alpha$ in glutamate-induced apoptosis in a differentiated neuronal cell line. Brain Res 2006; 1122: 201-8.

10 Taoufik E, Petit E, Divoux D, Tseveleki V, Mengozzi M, Roberts ML, et al. TNF receptor I sensitizes neurons to erythropoietin- and VEGFmediated neuroprotection after ischemic and excitotoxic injury. Proc Natl Acad Sci U S A 2008; 105: 6185-90.

11 Zi WJ, Shuai J. Plasma D-dimer levels are associated with stroke subtypes and infarction volume in patients with acute ischemic stroke. PLoS One 2014; 9: e86465.

12 Isgrò MA, Bottoni P, Scatena R. Neuron-specific enolase as a biomarker: biochemical and clinical aspects. Scatena R, editor. Adv Exp Med Biol 2015; 867: 125-43.

13 Pandey A, Shrivastava AK, Saxena K. Neuron specific enolase and c-reactive protein levels in stroke and its subtypes: correlation with degree of disability. Neurochem Res 2014; 39: 1426-32.

14 Singh HV, Pandey A, Shrivastava AK, Raizada A, Singh SK, Singh N. Prognostic value of neuron specific enolase and IL-10 in ischemic stroke and its correlation with degree of neurological deficit. Clin Chim Acta 2013; 419: 136-8.

15 Rizvi I, Islam N, Ullah E, Beg M, Akhtar N, Zaheer S. Correlation between serum neuron specific enolase and functional neurological outcome in patients of acute ischemic stroke. Ann Indian Acad Neurol 2013; 16: 504.

16 Lu K, Xu X, Cui S, Wang F, Zhang B, Zhao Y. Serum neuron specific enolase level as a predictor of prognosis in acute ischemic stroke patients after intravenous thrombolysis. J Neurol Sci 2015; 359: 202-6.

17 Yang XY, Gao S, Ding J, Chen Y, Zhou XS, Wang JE. Plasma D-dimer predicts short-term poor outcome after acute ischemic stroke. PLoS One 2014; 9: e89756

18 Pekar T, Stojakovic T, Haas J, Simmet NE, Scharnagl H, Gattringer T, et al. Plasma neutrophil gelatinase-associated lipocalin and functional outcome in ischemic stroke. J Neurol Sci 2013; 333: e171.

19 Matsuo R, Ago T, Hata J, Wakisaka Y, Kuroda J, Kuwashiro T, et al. Plasma C-reactive protein and clinical outcomes after acute ischemic stroke: a prospective observational study. PLoS One 2016; 11: e0156790.

20 Adams HP, Bendixen BH, Kappelle ப, Biller J, Love BB, Gordon DL, et al. Classification of subtype of acute ischemic stroke. Definitions for use in a multicenter clinical trial. TOAST. Trial of Org 10172 in Acute Stroke Treatment. Stroke 1993; 24: 35-41.

21 Hosmer DW, Lemeshow S. Applied logistic regression. Vol. 2nd. 2000

22 Den Hertog HM, Van Rossum JA, Van Der Worp HB, Van Gemert HMA, De Jonge $\mathrm{R}$, Koudstaal PJ, et al. C-reactive protein in the very early phase of acute ischemic stroke: association with poor outcome and death. J Neurol 2009; 256: 2003-8.

23 Seo WK, Seok HY, Kim JH, Park MH, Yu SW, Oh K, et al. C-reactive protein is a predictor of early neurologic deterioration in acute ischemic stroke. J Stroke Cerebrovasc Dis 2012; 21: 181-6.

24 Wang G, Weng YC, Han X, Whaley JD, McCrae KR, Chou WH. Lipocalin-2 released in response to cerebral ischaemia mediates reperfusion injury in mice. J Cell Mol Med 2015; 19: 1637-45.

25 Yan L, Borregaard N, Kjeldsen L, Moses MA. The high molecular weight urinary matrix metalloproteinase (MMP) activity is a complex of gelatinase B/MMP-9 and neutrophil gelatinase-associated lipocalin (NGAL). J Biol Chem 2001; 276: 37258-65.

26 Wen Hai Chou YCW. Neutrophil gelatinase-associated lipocalin and matrix metalloproteinase- 9 as potential biomarkers for stroke: a pilot study. J Neurol Neurophysiol 2015; 6: 6-11

27 Hochmeister S, Engel O, Adzemovic MZ, Pekar T, Kendlbacher P, Zeitelhofer M, et al. Lipocalin-2 as an infection-related biomarker to predict clinical outcome in ischemic stroke. PLoS One 2016; 11: e0154797.

28 Kawata K, Liu CY, Merkel SF, Ramirez SH, Tierney RT, Langford D. Blood biomarkers for brain injury: what are we measuring? Neurosci Biobehav Rev 2016; 68: 460-73.

29 Hrytsiuk T. Serum level of neuron-specific enolase in patients with past ischemic stroke. Arch Clin Med 2016; 22: 1-7.

30 Singh HV, Pandey A, Shrivastava AK, Raizada A, Singh SK, Singh N. Prognostic value of neuron specific enolase and IL-10 in ischemic stroke and its correlation with degree of neurological deficit. Clin Chim Acta 2013; 419: 136-8.

31 Yang XY, Gao S, Ding J, Chen Y, Zhou XS, Wang JE. Plasma D-dimer predicts short-term poor outcome after acute ischemic stroke. PLoS One 2014; 9: e89756.

32 Abd-Elhamid Y, Tork M, Abdulghani M. Prognostic value of D-dimer measurement in patients with acute ischemic stroke. Egypt $\mathrm{J}$ Neurol Psychiatry Neurosurg 2016; 53: 146-50.

33 Bisoendial RJ, Kastelein JJP, Levels JHM, Zwaginga JJ, van den Bogaard B, Reitsma $\mathrm{PH}$, et al. Activation of inflammation and coagulation after infusion of C-reactive protein in humans. Circ Res 2005; 96: 714 LP-716.

34 Csala M, Léránt I, Bánhegyi G, Kardon T, Puskás F, Mucha I, et al. Prostaglandin-independent stimulation of interleukin- 6 production by fibrinogen degradation product $D$ in perfused murine liver. Scand J Immunol 1998; 48: 269-71.

35 Ceulemans AG, Zgavc T, Kooijman R, Hachimi-Idrissi S, Sarre $S$, Michotte $Y$. The dual role of the neuroinflammatory response after ischemic stroke: modulatory effects of hypothermia. J Neuroinflammation 2010; 7: 74.

36 Sriram K, O'Callaghan JP. Divergent roles for tumor necrosis factoralpha in the brain. J Neuroimmune Pharmacol 2007; 2: 140-53.

37 Huang $H$, Huang Q, Wang F, Milner R, Li L. Cerebral ischemiainduced angiogenesis is dependent on tumor necrosis factor receptor 1-mediated upregulation of $\alpha 5 \beta 1$ and $\alpha \mathrm{V} \beta 3$ integrins. J Neuroinflammation 2016; 13: 227.

38 Svensson EH, Söderholm M, Abul-Kasim K, Engström G. Tumor necrosis factor receptor 1 and 2 are associated with risk of intracerebral hemorrhage. Stroke 2017; 48: 2710-5.

39 Greisenegger S, Segal HC, Burgess Al, Poole DL, Mehta Z, Rothwell PM. Biomarkers and mortality after TIA and minor ischemic stroke: population-based study. Stroke 2015; 46: 659-66. 